\title{
The Distribution of the Time of Ruin, the Surplus Immediately before Ruin and Deficit at Ruin under Two Sided Risk Renewal Process
}

\author{
Joseph Justin Rebello ${ }^{*}$, K. K. Thampi ${ }^{2}$ \\ ${ }^{1}$ Department of Statistics, AC, Mahatma Gandhi University, Kerala, India \\ ${ }^{2}$ Department of Statistics, SNMC, Mahatma Gandhi University, Kerala, India \\ Email: ^jj_rebello@yahoo.co.in
}

How to cite this paper: Rebello, J.J. and Thampi, K.K. (2017) The Distribution of the Time of Ruin, the Surplus Immediately before Ruin and Deficit at Ruin under Two Sided Risk Renewal Process. Journal of Mathematical Finance, 7, 624-632. https://doi.org/10.4236/jmf.2017.73032

Received: February 9, 2017

Accepted: July 14, 2017

Published: July 17, 2017

Copyright $\odot 2017$ by authors and Scientific Research Publishing Inc. This work is licensed under the Creative Commons Attribution International License (CC BY 4.0).

http://creativecommons.org/licenses/by/4.0/

\begin{abstract}
In the renewal risk theory, the study of two sided jumps has been attracted by many researchers since its introduction. After the development of the distribution of modified inter time claim occurrence, the explicit expressions for ruin theory components in the literature under some assumptions, in this work, we examine probability density of the time of ruin, surplus immediately before ruin and deficit at ruin respectively under two sided risk process using some basic assumptions. Explicit expressions for distribution of interest are being derived.
\end{abstract}

\section{Keywords}

Two Sided Jumps, Renewal Risk Process, Random Gain, Lindley Distribution, Erlangian Distribution

\section{Introduction}

The ruin problems and its analysis have been studied extensively by many authors ever since its inception. In the second half of 1900's Dickson et.al, Gerber et al. etc. have made a remarkable contribution to risk process both renewal and classical as well. Recently more works have been carried down in somewhat new approach in risk renewal process, so-called two sided jump process. Gerber and Shiu [1] have studied the joint density of the time of ruin, the surplus immediately before ruin and the deficit at ruin in the classical model of collective risk theory. Dufrence and Gerber [2] studied the joint density of function of the surplus immediately prior to ruin and deficit at ruin, later on, by adding one more quantity, the time of ruin. Their results have triggered a widespread research on this topic. For recent results in this area, we refer to Dickson and Hipp [3] [4], 
Cheng and Tang [5], Gerber and Shiu [6] [7] [8], Li and Garrido [9] [10], Pitts and Polis [11] that have generalized the results for phase type distributions and for sub exponential distributions.

The success of an insurance company is not only due to earnings in its conventional business but also due to intelligent investments of the money at its hand. Eventually risk invest can breed danger at the period when the market value of the assets is low and the company will not be able to recover from the loss because of market fluctuations. Therefore it is important to have a look on investments upon risk or risk free strategies. The investment of the money and settling down of the claim amount lead to a new branch of risk process called two sided risk process. More recently risk theory researches in two sided jumps field have got a remarkable attention. Substantial amount of works has been devoted to find the various ruin probability components under two sided risk model. For research on this kind of model, we refer to Perry, Stadje and Zacks [12], Cai and Yang [13], Yang and Zhimin [14], Jacobson [15], Xing, Zhang and Jiang [16], Zhang, Yang and Li [17], Albrecher, Gerber and Yang [18], Yang and Zhang [19], Dong and Liu [20] and Korolev, Chertok and Korchagin [21]. Although many published works concern with the multi dimensional risk process, the study is still in developing stage. Many problems are still far from the solution. The main reason of this is the complexity of mathematics involved in the problem.

The current paper aims at studying risk model with two sided jumps under some valid assumptions. In this paper we aim to derive a simple and unified expression for the densities of the time of ruin, the surplus immediately before ruin and deficit at ruin under two sided renewal risk process. The rest of the paper is organized as follows; In Section 2 we shall introduce some preliminary knowledge and focus on the main problem. In Section 3 we derive the densities under the condition suggested followed by the concluding remark.

\section{Model and Assumptions}

\subsection{Risk Model with Two Sided Jumps}

Stochastic process with two sided jumps has captured a lot of attention in recent times. Various first crossing times, two sided passage problems, ascending/ descending and some other related quantities have been studied under different model. The classical or Sparre Anderson renewal model consider only one sided rather the claim amount along with number of claims under some basic assumptions. The premium amount assumed to be constant and the respective random variables such as number of claims, claim size are also assumed to be independent to each other. In two sided jump problems we let the surplus amount at any point of time to be a function of more random variables. In other words we deposit or invest the surplus amount in some other profit making portfolio which breeds one more random variable preferably in positive direction. Apart from negatively moving claim size variable we thus having a one more positively moving random gain variable. This leads to a new kind of risk model termed as 
risk model with two sided jumps. This model can be extended to risk model with multi dimensional moves by incorporating more significant variables.

\subsection{Model}

Following the idea of Dong and Liu [20] we consider the surplus process as

$$
R(t)=u+p t+\sum_{i=1}^{M(t)} X_{i}-\sum_{j=1}^{N(t)} Y_{j}, t \geq 0
$$

where $u \geq 0$, the initial surplus, $p>0$, the constant premium rate, $\sum_{i=1}^{M(t)} X_{i}$ Compound Poisson process with intensity $\lambda$ representing the total random gain

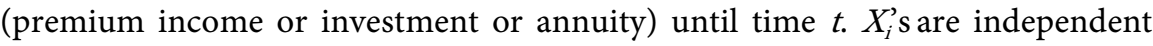
and identically distributed random variables with common density $\psi$ and mean $\mu_{x}$. Here we assume that $X$ follows an Erlangian $(2, \beta)$ and Laplace transforms of $\psi$ be $\bar{\psi}(s)=\int_{0}^{\infty} \mathrm{e}^{-s x} \psi(x) \mathrm{d} x$. Therefore

$$
\psi(x)=\beta^{2} x \mathrm{e}^{-\beta x}, x>0, \beta>0
$$

and $\widehat{\psi}(s)=\frac{\beta^{2}}{(s+\beta)^{2}}$, whereas $\sum_{j=1}^{N(t)} Y_{j}$ is accumulated claim process and $\{N(t)\}$ is a Renewal process representing the number of claims up to time $t$ with inter claim $\left\{H_{i}\right\}$. The $H_{i}^{\text {'s }}$ are i.i.d random variables with common density $\xi$ and Laplace transform $\bar{\xi}(s)=\int_{0}^{\infty} \mathrm{e}^{-s h} \xi(h) \mathrm{d} h$. The claim sizes $\left\{Y_{j}\right\}$ are positive random variables with a common distribution function $Q$, density $q$ and mean $\mu_{y}$ Laplace transform $\hat{q}(s)=\int_{0}^{\infty} \mathrm{e}^{-s y} q(y) \mathrm{d} y$. Here we assume that $Y$ follows Lindley distribution with parameter $\theta$. Then, $q(y)=\frac{\theta^{2}}{1+\theta}(1+y) \mathrm{e}^{-\theta y}, \theta>0$, [22] and $\hat{q}(s)=\frac{\theta^{2}(1+s+\theta)}{(1+\theta)(s+\theta)^{2}}$.

Also we assume that $\left\{X_{i}\right\},\left\{Y_{j}\right\},\{M(t)\}$ and $\{N(t)\}$ are mutually independent. For ensuring a positive security loading condition, we take $\left(p+\lambda \mu_{x}\right) \cdot E(H)>\mu_{y}$.

Let $T=\inf \{t \geq 0, R(t)<0\}$ be the ruin time, $R\left(T_{-}\right)$be the surplus immediately before ruin, namely undershoot and $|R(T)|$ be the deficit at ruin, termed as overshoot, again we define the probability of ruin

$$
\psi(u)=P[R(T)<0 / R(0)=u], u \geq 0 .
$$

For $\delta>0$, due to Gerber and Shiu [1]

$$
\Phi(u)=E\left[\mathrm{e}^{-(\delta T)} w\left(R\left(T_{-}\right),|R(T)|\right) I(T<\infty) / R(0)=u\right], u \geq 0
$$

where $w(x, y)$ is a non-negative measurable function on $[0, \infty) \times[0, \infty)$ and $I($.$) is an indicator function. When w(x, y)=1$ and $\delta=0, \Phi(u)$ becomes the probability of ruin, 


$$
\psi(u)=E[I(T<\infty) / R(0)=u]=P[T<\infty / R(0)=u], u \geq 0 .
$$

Again, Let $T_{n}=\sum_{i=1}^{n} H_{i}$ be the time when $\mathrm{n}^{\text {th }}$ claim occurs, $T_{0}=0$. Since ruin only occurs at the epochs where claims occur, then we define the discrete time process $\tilde{R}=\left\{\tilde{R}_{n}, n=0,1,2, \cdots\right\}$ and $\tilde{R}_{0}=0$

Again $\tilde{R}_{n}=R\left(T_{n}\right)$ denotes the surplus immediately after the $\mathrm{n}^{\text {th }}$ claim.

Now,

$$
\tilde{R}_{n}=u+p T_{n}+\sum_{i=1}^{M\left(T_{n}\right)} X_{i}-\sum_{k=1}^{n} Y_{k}=u+p \tilde{T}_{n}-\sum_{k=1}^{n} Y_{k}
$$

where $\tilde{T}_{n}=T_{n}+\sum_{i=1}^{M\left(T_{n}\right)} X_{i} / p$ with $\tilde{T}_{0}=0$ which corresponds to the Sparre Anderson risk model [23]. Thus

$$
\bar{R}(t)=u+p t-\sum_{i=1}^{\bar{N}(t)} Y_{i}
$$

where the initial surplus $\mathrm{u}$ and the claim size $Y_{i}$ are exactly the same as those in model (1). The counting number process $\bar{N}(t)$ denotes the number of claims up to time $\mathrm{t}$ with the modified inter claim times $Z_{i}=\tilde{T}_{i}-\tilde{T}_{i-1}$. Clearly $Z_{i}$ are i.i.d random variables with a common density $k$. (say)

\section{Derivation of the Density Functions}

In this model we assume the initial inter claim time, $H \sim \exp (\alpha)$, the number of claims $M(T)$ follows Poisson $(\lambda)$, the random gain $X \sim \operatorname{Erlang}(2, \beta)$ and finally we propose the claim amount $Y$ follows Lindley distribution with parameter $\theta$.

For $u \geq 0$, let $f(x, y, t / u)$ represents joint probability density function of $R\left(T_{-}\right),|R(T)|$ and $T$, then

$$
\int_{0}^{\infty} \int_{0}^{\infty} \int_{0}^{\infty} f(x, y, t / u) \mathrm{d} x \mathrm{~d} y \mathrm{~d} t=P(T<\infty / R(0)=u)=\psi(u)
$$

taking $T$ as time of ruin.

We let that $x>u+p t, f(x, y, t / u)=0$, or $x \leq u+p t, f(x, y, t / u)=1$ and

$$
f(u+p t, y, t / u) \mathrm{d} x \mathrm{~d} y \mathrm{~d} t=k(t) \cdot 1 \cdot f(u+p t+y) \mathrm{d} y \mathrm{~d} t
$$

where $k(t)$ is the linear combination of exponential distribution (Rebello \& Thampi, [24]).

$$
k(t)=a_{1} \mathrm{e}^{-R_{1} t}+a_{2} \mathrm{e}^{-R_{2} t}+a_{3} \mathrm{e}^{-R_{3} t}, t>0
$$

$a_{1}, a_{2}, a_{3}, R_{1}, R_{2}$ and $R_{3}$ are suitably chosen constants.

It is known, using $P(X \cup Y)=P(X) \cdot P(Y / X)$, that $f(x, y, t / u)$ is the jpdf of $R\left(T_{-}\right)$and $T$ at the point $(x, t)$ multiplied by the conditional probability density function of $|R(T)|$ at $\mathrm{y}$ given that $R\left(T_{-}\right)=x$ and $T=t$.

Since the conditional probability $\left|R\left(T_{-}\right)\right|$does not depend on $t$ we have

$$
\frac{\gamma(x+y)}{\int_{0}^{\infty} \gamma(x+y) \mathrm{d} x}=\frac{\gamma(x+y)}{\bar{\Upsilon}(x)} \text { (Gerber and Shiu, [1]) }
$$


hence

$$
\begin{aligned}
& f(x, y, t / u)=P\left(R\left(T_{-}\right) \cap T\right) \cdot P\left(|R(T)| / R\left(T_{-}\right) \cap T\right) \\
& \Rightarrow f(x, y, t / u)=\int_{0}^{\infty} f(x, z, t / u) \mathrm{d} z \frac{\gamma(x+y)}{\bar{\Upsilon}(x)}
\end{aligned}
$$

Define

$$
p(x, y / u)=\int_{0}^{\infty} \mathrm{e}^{-\delta t} f(x, y, t / u) \mathrm{d} t
$$

where $\delta$ can be considered as a force of interest, as a dummy variable, and $\chi(x / u)=\int_{0}^{\infty} p(x, y / u) \mathrm{d} y$. multiplying (2) by $\mathrm{e}^{-\delta t}$ and integrating with respect to t. i.e.,

$$
\begin{gathered}
\int_{0}^{\infty} \mathrm{e}^{-\delta t} f(x, y, t / u) \mathrm{d} t=\int_{0}^{\infty} \mathrm{e}^{-\delta t} \int_{0}^{\infty} f(x, z, t / u) \mathrm{d} z \cdot \frac{\gamma(x+y)}{\bar{\Upsilon}(x)} \mathrm{d} t \\
p(x, y / u)=\chi(x / u) \cdot \frac{\gamma(x+y)}{\bar{\Upsilon}(x)}
\end{gathered}
$$

Hence $g(y)=\int_{0}^{\infty} p(x, y / u) \mathrm{d} x$ i.e., $g(y)=\int_{0}^{\infty} \int_{0}^{\infty} \mathrm{e}^{-\delta t} f(x, y, t / 0) \mathrm{d} t \mathrm{~d} x$.

Using the definition of martingale; upon the stochastic process $\{R(t)\}_{t \geq 0}$ with stationary and independent increments, the process $\left\{\mathrm{e}^{-\delta t+\xi R(t)}\right\}_{t \geq 0}$ is a martingale iff for each $t>0$ its expectation at time $t$ is equal to its initial value (where $\xi$ is a constant).

$$
\text { i.e., iff } E\left(\mathrm{e}^{-\delta t+\xi R(t)} / R(0)=u\right)=E\left(\mathrm{e}^{\xi u}\right)=\mathrm{e}^{\xi u}
$$

Define $\bar{\chi}$ be the Laplace transform of a function $\chi(x), x>0$,

$$
\bar{\chi}(\xi)=\int_{0}^{\infty} \mathrm{e}^{-\xi x} \chi(x) \mathrm{d} x
$$

Then LHS of (3)

$$
\begin{aligned}
& R\left(\mathrm{e}^{-\delta t+\xi R(t)} / R(0)=u\right) \\
& =E\left(\mathrm{e}^{-\delta t+\xi\left\{U+p t-\sum_{i=1}^{\bar{N}(t)} X_{i}\right\}}\right)=E\left(\mathrm{e}^{-\delta t} \cdot \mathrm{e}^{\xi u} \cdot \mathrm{e}^{\xi p t} \cdot \mathrm{e}^{-\sum_{i=1}^{\bar{N}(t)} X_{i}}\right) \\
& =\exp \{-\delta t+\xi u+\xi p t+\lambda t(\bar{\chi}(\xi)-1)\}
\end{aligned}
$$

(3) Implies $-\delta+\xi p+\lambda(\bar{\chi}(\xi)-1)=0$.

It tends to $\infty$ as $\xi \rightarrow \infty$ and takes the value $-\delta$ at $\xi=0$. thus it has a unique non-negative root, $\rho$ hence $\left\{\mathrm{e}^{-\delta t+\rho R(t)}\right\}_{t \geq 0}$ is a martingale.

For $x>u=R(0)$, let $T_{x}=\inf \{t / R(t)=x\}$ be the first time when the surplus reaches the level $\mathrm{x}$.

Then $R(t) \leq x, 0 \leq t \leq T_{x}$ and using optimal stopping theorem to $\left\{\mathrm{e}^{-\delta t+\rho R(t)}\right\}_{t \geq 0}$, we have 


$$
\mathrm{e}^{\rho u}=E\left(\mathrm{e}^{-\delta T_{x}+\rho R\left(T_{x}\right)} / R(0)=u\right)=\mathrm{e}^{\rho x} \cdot E\left(\mathrm{e}^{-\delta T_{x}} / R(0)=u\right)
$$

Or

$$
E\left(\mathrm{e}^{-\delta T_{x}} / R(0)=u\right)=\mathrm{e}^{-\rho(x-u)}
$$

Using the idea of Gerber and Shiu [1],

For $x>u$, let $\pi(t ; u, x) ; t>0$ denote the pdf of the random variable $T$.

Hence (4) implies $\int_{0}^{\infty} \mathrm{e}^{-\delta t} \pi(t ; u, x) \mathrm{d} t=\mathrm{e}^{-\rho(x-u)}$. The differential $\pi(t ; u, x) \mathrm{d} t$ is the probability that the surplus process meets the level $x$ first time between $(t, t+$ $\mathrm{d} t$ ) provides the surplus cannot reach $x$ before $t=\frac{x-u}{p}$. Hence for $t<\frac{x-u}{p} ; \pi(t ; u, x)=0$ and the density function $\pi(t ; u, x)$ at $t=\frac{x-u}{p}$ equals $\frac{a_{1}}{R_{1}} \cdot\left(1-\mathrm{e}^{-R_{1}(x-u) / p}\right)+\frac{a_{2}}{R_{2}} \cdot\left(1-\mathrm{e}^{-R_{2}(x-u) / p}\right)+\frac{a_{3}}{R_{3}} \cdot\left(1-\mathrm{e}^{-R_{3}(x-u) / p}\right)$ or $=\sum_{i=1}^{3} \frac{a_{i}}{R_{i}} \cdot\left(1-\mathrm{e}^{-R_{i}(x-u) / p}\right)$.

Using duality $\pi(t ; u, x)=\tilde{\pi}(t ; u, x)$, Now the differential $f(x, y, t / u) \mathrm{d} x \mathrm{~d} y \mathrm{~d} t$ can be interpreted as the probability of the event that ruin does not take place by the time $\mathrm{t}$, that the surplus process meets the level $x$ between $(t, t+\mathrm{d} t)$, but does not attain the level $x+\mathrm{d} x$ owing a claim within $\mathrm{d} x / p$ time units after $T_{x}$ and that the size of this claim is $x+y$.

$$
\therefore f(x, y, t / u) \mathrm{d} x \mathrm{~d} y \mathrm{~d} t=\tilde{\pi}(t ; u, x) \mathrm{d} t \cdot \lambda \frac{\mathrm{d} x}{p} \cdot \gamma(x+y) \mathrm{d} y
$$

$\mathrm{Or}$

$$
f(x, y, t / u)=\frac{\lambda}{p} \cdot \sum_{i=1}^{3} \frac{a_{i}}{R_{i}} \cdot\left(1-\mathrm{e}^{-R_{i}(x-u) / p}\right) \cdot \frac{\theta^{2}}{1+\theta}(1+x+y) \mathrm{e}^{-\theta(x+y)}
$$

using (4), if we multiply (5) by $\mathrm{e}^{-\delta t} \mathrm{~d} t$ and integrate from $t=0$ to $t=\infty$ and apply(4), we have

$$
p(x, y / u)=\frac{\lambda}{p} \cdot \mathrm{e}^{-\rho x} \cdot \frac{\theta^{2}}{1+\theta}(1+x+y) \mathrm{e}^{-\theta(x+y)}
$$

Or

$$
\chi(x / 0)=\frac{\lambda}{p} \cdot \mathrm{e}^{-\theta x}\left(1+\frac{\theta x}{\theta+1}\right) \cdot \mathrm{e}^{-\rho x}
$$

and

$$
g(y)=\frac{\lambda}{p} \cdot \int_{0}^{\infty} \frac{\theta^{2}}{1+\theta} \mathrm{e}^{-\theta(x+y)}(1+x+y) \cdot \mathrm{e}^{-\rho x} \mathrm{~d} x
$$

\section{Corollary}

By distinguishing whether or not ruin occurs at the first time when the surplus falls below the initial value $\mathrm{u}$, we have 


$$
p(x, y / u)=\int_{0}^{u} p(x, y / u-z) \cdot g(z) \mathrm{d} z+p(x-u, y+u / 0), 0<u<x
$$

Using (7)

$$
\begin{gathered}
p(x, y / u)=\frac{\lambda}{p} \mathrm{e}^{-\rho x} \gamma(x+y) \\
p(x-u, y+u / 0)=\frac{\lambda}{p} \mathrm{e}^{-\rho(x-u)} \gamma(x+y)=p(x, y / 0) \cdot \mathrm{e}^{\rho u} \\
\therefore(7) \Rightarrow p(x, y / u)=\int_{0}^{u} p(x, y / u-z) \cdot g(z) \mathrm{d} z+p(x, y / 0) \cdot \mathrm{e}^{\rho u} \cdot I(u<x)
\end{gathered}
$$

where $I(u<x)$ is an indicator function. Integrating (8) with respect to y, we

have $\chi(x / u)=\int_{0}^{u} \chi(x / u-z) \cdot g(z) \mathrm{d} z+\chi(x / 0) \cdot \mathrm{e}^{\rho u} \cdot I(u<x)$, defining

$$
\varsigma(u)=\int_{0}^{u} \varsigma(u-z) g(z) \mathrm{d} z+\mathrm{e}^{\rho u} I(u<x)
$$

then

$$
\chi(x / u)=\chi(x / 0) \varsigma(u)
$$

\section{Concluding Remarks}

This paper is prepared to learn the insurance process under two sided jump risk renewal processes. We investigated a reformulated insurance process in which random gain and claim amount are all uncertain renewal processes. This paper derives the distribution of time of ruin, deficit at ruin, surplus amount $t$ before ruin under two sided risk theory setup. The explicit expressions for the same are being derived. The application of other feasible distributions may be considered as a scope of further study.

\section{Acknowledgements}

The authors would like to thank the anonymous referee for helpful comments and suggestions which improved the earlier version of the paper. This research was supported by University Grants Commission of India.

\section{References}

[1] Gerber, H.U. and Shiu, E.S.W. (1997) The Joint Distribution of Time of the Ruin, the Surplus Immediately before Ruin and the Deficit at Ruin. Insurance: Mathematics and Economics, 21, 129-137. https://doi.org/10.1016/S0167-6687(97)00027-9

[2] Dufrense, F. and Gerber, U. (1988) The Probability and Severity of Ruin for Combinations of Exponential Claim Amount Distributions and Their Translations. Insurance: Mathematics and Economics, 7, 75-80. https://doi.org/10.1016/0167-6687(88)90100-X

[3] Dickson, D.C.M. and Hipp, C. (2000) Ruin Problems for Phase Type(2) Risk Process. Scandinavian Acturial Journal, 147-167.

[4] Dickson, D.C.M. and Hipp, C. (2001) On the Time to Ruin for Erlang(2) Risk Proc- 
ess. Insurance: Mathematics and Economics, 29, 333-344.

https://doi.org/10.1016/S0167-6687(01)00091-9

[5] Chang, V. and Tang, Q. (2003) Moments of the Surplus before Ruin and the Feficit at Ruin in the Erlang (2) Risk Process. North American Actuarial Journal, 7, 1-12. https://doi.org/10.1080/10920277.2003.10596073

[6] Gerber, H.U. and Shiu, E.S.W. (1998) On the Time Value of Ruin. North American Actuarial Journal, 2148-2178.

[7] Gerber, H.U. and Shiu, E.S.W. (2003) Discussion on Moments of the Surplus before Ruin and Deficit at the Ruin in the Erlang(2) Risk Process. North American Actuarial Journal, 7, 117-119. https://doi.org/10.1080/10920277.2003.10596110

[8] Gerber, H.U. and Shiu, E.S.W. (2003) Comments on Moments of the Surplus before Ruin and Deficit at the Ruin in the Erlang(2) Risk Process. North American Actuarial Journal, 9, 49-84. https://doi.org/10.1080/10920277.2005.10596197

[9] Li, S.M. and Garrido, J. (2004) On Ruin for the Erlang(n) Risk Process. Insurance: Mathematics and Economics, 34, 391-408. https://doi.org/10.1016/j.insmatheco.2004.01.002

[10] Li, S.M. and Garrido, J. (2005) On Ruin for the Erlang(n) Risk Process. Advances in Applied Probability, 35, 836-856. https://doi.org/10.1017/S0001867800000501

[11] Pitts, S.M. and Politis, K. (2007) The Joint Density of the Surplus before and after Ruin in the Sparre-Anderson Risk Model. Applied Probability Trust, 44, 695-712. https://doi.org/10.1017/S0021900200003375

[12] Perry, D., Stadje, W. and Zacks, S. (2002) First Exit Times for Compound Poisson Process for Some Types of Positive and Negative Jumps. Stochastic Models, 18, 139157. https://doi.org/10.1081/STM-120002778

[13] Cai, J. and Yang, H. (2005) Ruin in the Perturbed Compound Poisson Risk Process under Interest Force. Advances in Applied Probability, 37, 819-835.

https://doi.org/10.1017/S0001867800000495

[14] Yang, H. and Zhimin, Z. (2010) On a Discrete Risk Model with Two Sided Jumps. Journals Computational and Applied Mathematics, 234, 835-844. https://doi.org/10.1016/j.cam.2010.01.047

[15] Jacobson, M. (2005) The Time to Ruin for a Class of Markov Additive Risk Process with Two Sided Jumps. Advances in Applied Probability, 27, 963-992. https://doi.org/10.1017/S0001867800000628

[16] Xing, X., Zhang, W. and Jiang, Y. (2008) On the Time to Ruin and Deficit at Ruin in a Risk Model with Double Sided Jumps. Statistics and Probability Letters, 78, 2692 2699. https://doi.org/10.1016/j.spl.2008.03.034

[17] Zhang, Z., Yang, H. and Li, S. (2010) The Perturbed Compound Risk Model with Two Sided Jumps. Computational and Applied Mathematics, 80, 1773-1784. https://doi.org/10.1016/j.cam.2009.09.014

[18] Albretcher, H., Gerber, H.U. and Yang, H. (2010) A Direct Approach to the Discounted Penalty Functions. North American Actuarial Journal, 14, 420-447. https://doi.org/10.1080/10920277.2010.10597599

[19] Zhang, Z. and Yang, H. (2010) A Generalised Penalty Function in the SparreAnderson Risk Model with Two Sided Jumps. Statistics Probability and Letters, 80, 597-607. https://doi.org/10.1016/j.spl.2009.12.016

[20] Dong, H. and Liu, Z. (2013) The Ruin Problem in a Renewal Risk Model with Two Sided Jumps. Mathematical and Computer Modeling, 57, 800-811. https://doi.org/10.1016/j.mcm.2012.09.005

[21] Korelov, V.Y., Chertok, A.N. and Korchagin, A.Y. (2015) Modelling High Fre- 
quency Order Flow Imbalance by Functional Limit Theorems for Two Sided Risk Process. Applied Mathematics and Computation, 253, 224-241.

https://doi.org/10.1016/j.amc.2014.12.085

[22] Ghitany, M.E., Atich, B. and Nadarajah, S. (2008) Lindley Distribution and Its Applications. Mathematics and Computers in Simulation, 78, 493-506.

https://doi.org/10.1016/j.matcom.2007.06.007

[23] Anderson, E.S. (1957) On the Collective Theory of Risk in Case of Contagion between the Claims. Transactions on XVth International Congress of Actuaries, 2, 219-229.

[24] Rebello, J.J. and Thampi, K.K. (2017) Some Ruin Theory Components of Two Sided Jump Problems under Renewal Risk Process. International Mathematical Forum, 12, 311-325. https://doi.org/10.12988/imf.2017.611147

Submit or recommend next manuscript to SCIRP and we will provide best service for you:

Accepting pre-submission inquiries through Email, Facebook, LinkedIn, Twitter, etc. A wide selection of journals (inclusive of 9 subjects, more than 200 journals) Providing 24-hour high-quality service User-friendly online submission system Fair and swift peer-review system Efficient typesetting and proofreading procedure Display of the result of downloads and visits, as well as the number of cited articles Maximum dissemination of your research work

Submit your manuscript at: http://papersubmission.scirp.org/

Or contact jmf@scirp.org 